Notfall Rettungsmed

https://doi.org/10.1007/s10049-022-00992-3

Angenommen: 25. Januar 2022

(c) Der/die Autor(en) 2022

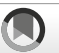

Check for
updates

\section{Medikamentengabe durch Notfallsanitäter}

\section{Eine Studie mit Blick auf die Ausbildung}

Hintergrund: Der Bundesverband der Ärztlichen Leiter Rettungsdienst (BV-ÄLRD) hat einen umfangreichen Katalog an Medikamenten für die Anwendung durch Notfallsanitäter vorgeschlagen. Wir evaluierten, wie oft Auszubildende zum Notfallsanitäter diese in ihrer Ausbildung applizierten. Methode: Wir befragten 71 Auszubildende zum Notfallsanitäter zur Anwendungshäufigkeit 30 verschiedener Medikamente. Die statistische Auswertung erfolgte mit SOFA (Paton-Simpson and Associates Ltd, USA). Die Ergebnisse sind als Mittelwert (Standardabweichung) dargestellt.

Ergebnisse: Adrenalin i.v. wurde 2,22 (6,67) Mal verabreicht. Atropin wurde bei Bradykardie 1,18 (1,97) Mal verabreicht. Benzodiazepine wurden präklinisch 7,27 $(10,28) \mathrm{Mal}$ angewendet. Inhalative Betamimetika wurden präklinisch 8,89 $(8,06) \mathrm{Mal}$, Ipratropiumbromid 3,70 (5,91) Mal, Kortikoide 3,79 $(5,32)$ Mal und Antihistaminika 1,82 $(4,34)$ Mal verabreicht. Ondansetron wurde innerklinisch 14,38 $(35,06)$ Mal angewendet. Präklinisch wurde Dimenhydrinat mit 8,14 $(10,14)$ Anwendungen am häufigsten verabreicht. Furosemid wurde präklinisch 6,06 $(9,45)$ Mal verabreicht. Heparin wurde präklinisch 12,00 (14,32) Mal, ASS 14,32 (14,29) Mal und Glyceroltrinitrat 12,25 (10,87) Mal verabreicht. Metamizol wurde präklinisch 8,59 (12,51) Mal, Ketamin 3,51 (5,23) Mal, Opiate/Opioide 10,72 $(16,18)$ Mal und Naloxon 0,20 $(0,67)$ Mal verabreicht. Glukose wurde präklinisch 7,38 $(6,67)$ Mal verabreicht.

Schlussfolgerung: Die Bewertung, für welche Medikamente eine ausreichende Expertise besteht, kann aufgrund fehlender Daten zu Lernkurven oder konsentierter Mindestzahlen nur vor dem Hintergrund klinischer Erfahrung erfolgen. Aus Sicht der Autoren besteht für die meisten untersuchten Medikamente eine ausreichende Expertise.

\title{
Schlüsselwörter
}

Notfallmedizin · Bundesverband Ärztlicher Leiter Rettungsdienst · Notfallmedikamente · Invasive Maßnahmen·Selbsteinschätzung

\section{Einleitung}

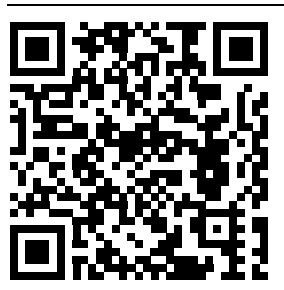

QR-Code scannen \& Beitrag online lesen
Am 01.01.2014 wurde mit dem Inkrafttreten des Notfallsanitätergesetzes (NotSanG) ein neues Berufsbild in den deutschen Rettungsdienst eingeführt. Der Gesetzgeber hat dabei explizit die Durchführung „auch invasiver Maßnahmen“ im Rahmen der Erstversorgungskompetenz sowie auch „heilkundlicher" Maßnahmen im Rahmen von vom ärztlichen Leiter vorgegebenen, überprüften und verantworteten Algorithmen durch Notfallsanitäterinnen und Notfallsanitäter vorgesehen (im Weiteren zur besseren Lesbarkeit gemeinsam einheitlich als Notfallsanitäter bezeichnet). Dabei wird vom Notfallsanitäter - wie von jedem anderen Behandler - erwartet, dass er die ergriffenen Maßnahmen erlernt hat und beherrscht [1]. Hierbei lässt sich für die 


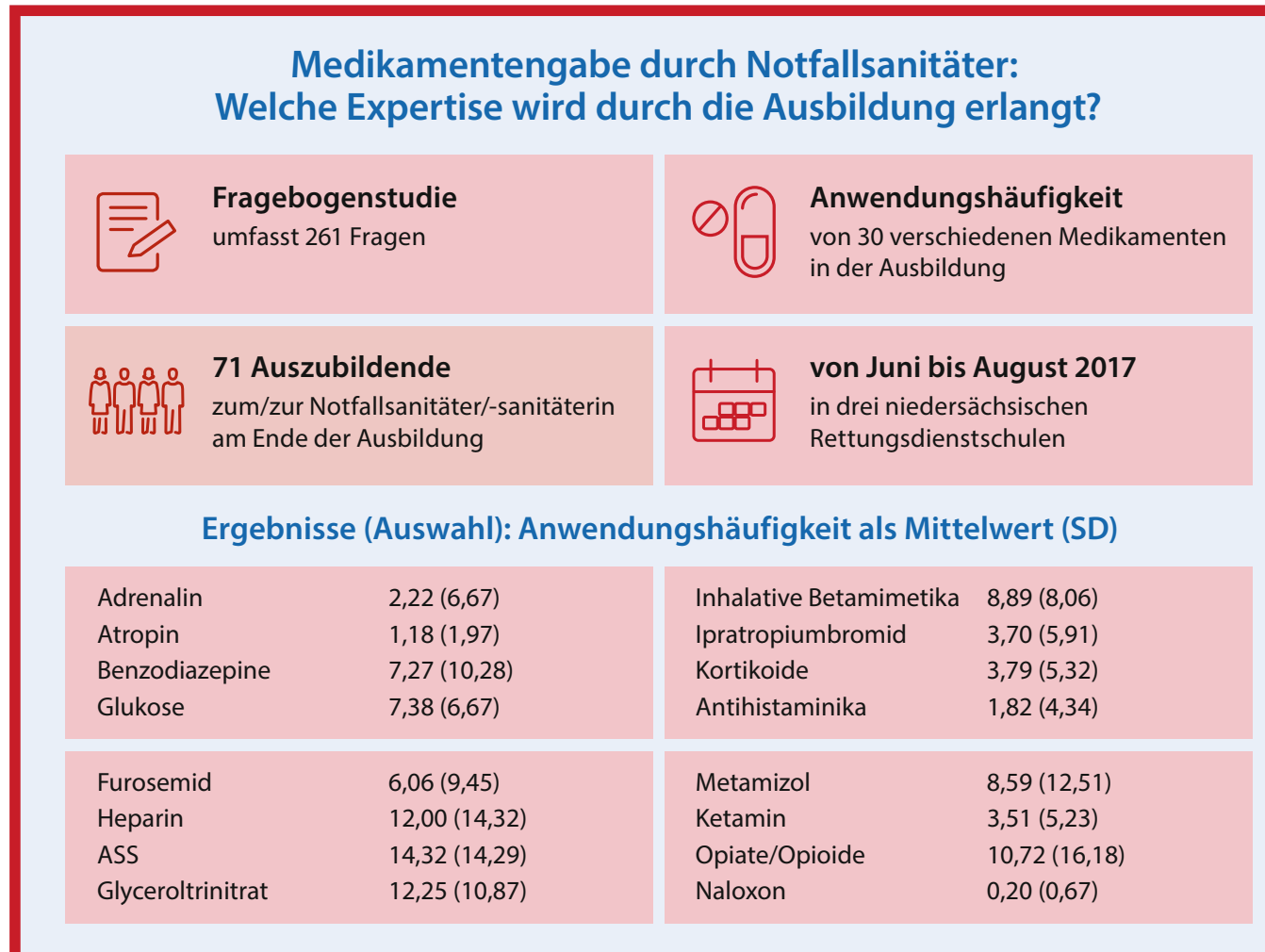

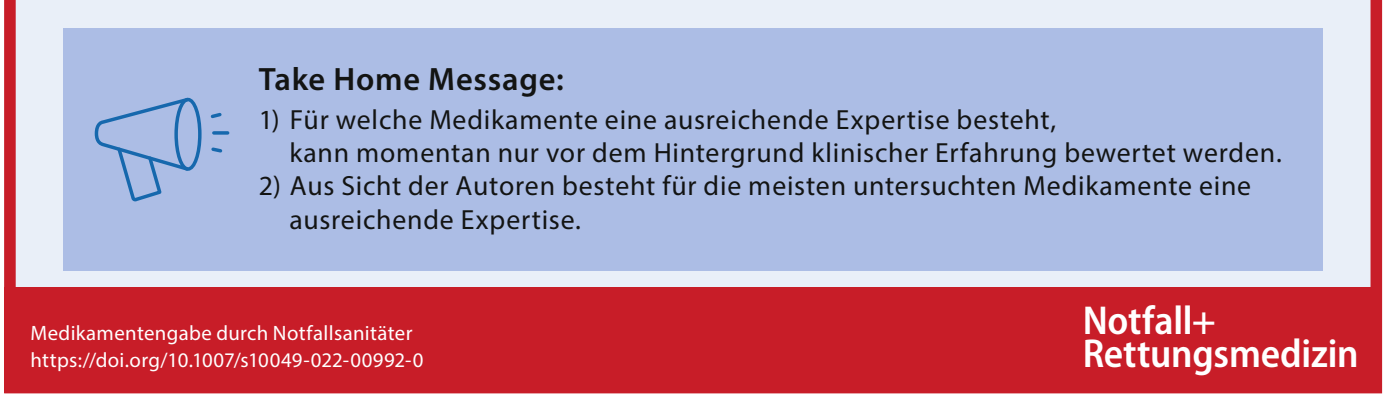

Abb. $1<$ Studiendesign und relevante Ergebnisse

einzelnen Maßnahmen jeweils eine Mindestzahl an Anwendungen beschreiben, die nötig sind, um diese zu erlernen. Für Medikamente ist dies schwieriger, da Studien zu Lernkurven bei der Verabreichung von Medikamenten fehlen. Zwar war die Verabreichung bestimmter Medikamente durch Rettungsdienstpersonal bereits vor Inkrafttreten des NotSanG in den meisten Rettungsdienstbereichen etabliert und durch Algorithmen geregelt, sodass hier bereits einige Erfahrung besteht, gleichwohl existieren nur wenige Studien zur Sicherheit und Effektivität der Medikamentengabe durch Rettungsdienstpersonal. Bei den vom Bundesverband der Ärztlichen Leiter Rettungsdienst (BV-ÄLRD) derzeit für eine Gabe durch Notfallsanitäter vorgeschlagenen Medikamenten besteht zudem eine Heterogenität in der Bewertung von Nutzen und Risiko der verschiedenen Substanzen. So sind einige Substanzen bei gegebener Indikation als eher unkritisch zu betrachten, wohingegen andere mit potenziell schweren Nebenwirkungen verbunden sind und daher in die Hand eines ausreichend erfahrenen Anwenders gehören. Die vorliegende Arbeit soll als eine erste Standortbestimmung darstellen, welche Erfahrung Notfallsanitäter im Rahmen ihrer Ausbildung bezogen auf die Verabreichung von Notfallmedikamenten erwerben. Dem soll eine Bewertung hinsichtlich Nutzen und Risiken der Substanzen gegenübergestellt werden.

Um zukünftigen Entwicklungen in der Medizin Rechnung zu tragen, wurde im Notfallsanitätergesetz nicht explizit vom Gesetzgeber festgelegt, welche Medikamente Notfallsanitäter konkret verabreichen sollen. Der Bundesverband Ärztli- cher Leiter Rettungsdienst (BV-ÄLRD) hat aus diesem Grund bereits 2014 eine Auswahl an Medikamenten vorgelegt, welche aus Sicht des Verbands für die Gabe durch Notfallsanitäter infrage kommen $[2,3]$. Die jeweils zuständigen Ärztlichen Leiter Rettungsdienst (ÄLRD) und die zuständigen Behörden wurden parallel dazu aufgefordert, die Verabreichung der einzelnen Medikamente im Rahmen von Algorithmen und Standard Operating Procedures zu konkretisieren und festzulegen, bei welchen "medizinischen Zustandsbildern" diese anzuwenden sind, in welcher Form und Dosierung sie zu applizieren sind und wann ein Notarzt nachzufordern ist.

In den einzelnen Bundesländern wurden daraufhin auf diesem Medikamentenkatalog basierende Algorithmen veröffentlicht, welche die Durchführung und Indikationsstellung der einzelnen Medikamen- 
Tab. 1 Im Rahmen der Ausbildung angewendete Medikamente und Anzahl der durchgeführten Medikamentengaben durch Notfallsanitäter unter besonderer Berücksichtigung des durch die Ärztlichen Leiter Rettungsdienst veröffentlichten Medikamentenkatalogs ${ }^{\mathrm{a}}[1]$

\begin{tabular}{|c|c|c|c|c|c|c|c|}
\hline \multirow[t]{2}{*}{ Medikament } & \multirow{2}{*}{$\begin{array}{l}\text { Erfragter Anwendungsbereich/ } \\
\text { Indikation }\end{array}$} & \multirow{2}{*}{$\begin{array}{l}\text { Indikation laut } \\
\text { BV-ÄLRD }\end{array}$} & \multicolumn{2}{|l|}{ Anzahl } & \multicolumn{3}{|c|}{ Bewertung } \\
\hline & & & $\begin{array}{l}\text { Mittelwert } \\
(n=71)\end{array}$ & $\begin{array}{l}\text { Standard- } \\
\text { abweichung } \\
(n=71)\end{array}$ & $\begin{array}{l}\text { Feste } \\
\text { Dosis }\end{array}$ & $\begin{array}{l}\text { Schwer- } \\
\text { wiegende } \\
\text { Komplikatio- } \\
\text { nen }\end{array}$ & $\begin{array}{l}\text { Vitale In- } \\
\text { dikation }\end{array}$ \\
\hline \multirow[t]{3}{*}{$\begin{array}{l}\text { Adrenalin intra- } \\
\text { venös }\end{array}$} & $\begin{array}{l}\text { Reanimation klinisch und präkli- } \\
\text { nisch }\end{array}$ & \multirow{3}{*}{$\begin{array}{l}\text { Reanimation, } \\
\text { Anaphylaxie, } \\
\text { Bradykardie }\end{array}$} & 9,76 & 13,31 & Ja & Nein & $\mathrm{Ja}$ \\
\hline & $\begin{array}{l}\text { Anaphylaxie klinisch und präkli- } \\
\text { nisch }\end{array}$ & & 0,28 & 0,70 & Nein & $\mathrm{Ja}$ & $\mathrm{Ja}$ \\
\hline & $\begin{array}{l}\text { Innerklinisch unabhängig von der } \\
\text { Indikation }\end{array}$ & & 2,22 & 6,67 & - & - & - \\
\hline \multirow[t]{2}{*}{$\begin{array}{l}\text { Adrenalin inhala- } \\
\text { tiv }\end{array}$} & $\begin{array}{l}\text { Bei Atemwegsschwellung oder } \\
\text { obstruktiver Atemnot klinisch und } \\
\text { präklinisch }\end{array}$ & \multirow[t]{2}{*}{$\begin{array}{l}\text { Asthma, Pseu- } \\
\text { dokrupp, Ana- } \\
\text { phylaxie }\end{array}$} & 0,73 & 1,03 & \multirow[t]{2}{*}{ Ja } & \multirow[t]{2}{*}{ Eher nein } & \multirow[t]{2}{*}{$\mathrm{Ja}$} \\
\hline & $\begin{array}{l}\text { Innerklinisch unabhängig von der } \\
\text { Indikation }\end{array}$ & & 0,21 & 0,77 & & & \\
\hline \multirow[t]{2}{*}{ Noradrenalin ${ }^{b}$} & Innerklinisch & \multirow[t]{2}{*}{ Keine Nennung } & 9,40 & 16,07 & \multirow[t]{2}{*}{ Nein } & \multirow[t]{2}{*}{ Eher ja } & \multirow[t]{2}{*}{ Ja } \\
\hline & Präklinisch & & 0,41 & 1,32 & & & \\
\hline \multirow[t]{2}{*}{ Akrinor $^{\circledR b}$} & Innerklinisch & \multirow[t]{2}{*}{ Keine Nennung } & 10,99 & 15,25 & \multirow[t]{2}{*}{ Nein } & \multirow[t]{2}{*}{ Nein } & \multirow[t]{2}{*}{$\mathrm{Ja}$} \\
\hline & Präklinisch & & 0,86 & 3,26 & & & \\
\hline \multirow[t]{4}{*}{ Amiodaron } & Bei Reanimation innerklinisch & \multirow{4}{*}{$\begin{array}{l}\text { Reanimation, } \\
\text { Ventrikuläre } \\
\text { Tachykardie }\end{array}$} & 0,45 & 1,07 & \multirow[t]{2}{*}{ Ja } & \multirow[t]{2}{*}{ Nein } & \multirow[t]{2}{*}{ Ja } \\
\hline & Bei Reanimation präklinisch & & 2,46 & 3,46 & & & \\
\hline & $\begin{array}{l}\text { Bei tachykarden Herzrhythmusstö- } \\
\text { rungen innerklinisch }\end{array}$ & & 0,72 & 2,05 & Nein & $\mathrm{Ja}$ & $\mathrm{Ja}$ \\
\hline & $\begin{array}{l}\text { Bei tachykarden Herzrhythmusstö- } \\
\text { rungen präklinisch }\end{array}$ & & 0,55 & 2,43 & & & \\
\hline \multirow[t]{3}{*}{ Atropin } & $\begin{array}{l}\text { Innerklinisch unabhängig von der } \\
\text { Indikation }\end{array}$ & \multirow{3}{*}{$\begin{array}{l}\text { Bradykardie, } \\
\text { Intoxikation mit } \\
\text { Alkylphospha- } \\
\text { ten }\end{array}$} & 10,75 & 11,91 & \multirow[t]{2}{*}{$\mathrm{Ja}$} & Eher nein & $\mathrm{Ja}$ \\
\hline & $\begin{array}{l}\text { Präklinisch bei bradykarden Herz- } \\
\text { rhythmusstörungen }\end{array}$ & & 1,18 & 1,97 & & & \\
\hline & $\begin{array}{l}\text { Bei Intoxikation mit Alkylphospha- } \\
\text { ten/Carbamaten }\end{array}$ & & 0,06 & 0,29 & Nein & Nein & $\mathrm{Ja}$ \\
\hline Benzodiazepine & Innerklinisch & Krampfanfall, & 8,20 & 11,36 & Nein & $\mathrm{Ja}$ & $\mathrm{Ja}$ \\
\hline & Präklinisch & $\begin{array}{l}\text { Status epilepti- } \\
\text { cus, Sedierung, } \\
\text { Erregungszu- } \\
\text { stände }\end{array}$ & 7,27 & 10,28 & & & \\
\hline Beta-2-Mimetika & $\begin{array}{l}\text { Inhalative Beta-2-Mimetika } \\
\text { innerklinisch }\end{array}$ & $\begin{array}{l}\text { Asthma, COPD, } \\
\text { Bronchitis }\end{array}$ & 6,07 & 11,06 & $\mathrm{Ja}$ & Nein & $\mathrm{Ja}$ \\
\hline & $\begin{array}{l}\text { Inhalative Beta-2-Mimetika } \\
\text { präklinischc }\end{array}$ & & 8,89 & 8,06 & & & \\
\hline & $\begin{array}{l}\text { Intravenöse Beta-2-Mimetika } \\
\text { innerklinischc }\end{array}$ & & 0,25 & 0,65 & $\mathrm{Ja}$ & Eher ja & \\
\hline & $\begin{array}{l}\text { Intravenöse Beta-2-Mimetika } \\
\text { präklinischc }\end{array}$ & & 0,68 & 1,74 & & & \\
\hline Ipratro- & Innerklinisch & Asthma, COPD, & 2,20 & 4,49 & $\mathrm{Ja}$ & Nein & $\mathrm{Ja}$ \\
\hline piumbromid & Präklinisch & Bronchitis & 3,70 & 5,91 & & & \\
\hline Kortikoide & Innerklinisch & Asthma, Allergie & 3,10 & 8,24 & $\mathrm{Ja}$ & Nein & Eher ja \\
\hline & Präklinisch & & 3,79 & 5,32 & & & \\
\hline Antihistaminika & Innerklinisch ${ }^{d}$ & Allergische & 6,62 & 29,37 & Ja & Nein & $\mathrm{Ja}$ \\
\hline & Präklinisch ${ }^{d}$ & Reaktion & 1,82 & 4,34 & & & \\
\hline
\end{tabular}




\begin{tabular}{|c|c|c|c|c|c|c|c|}
\hline \multirow[t]{2}{*}{ Medikament } & \multirow{2}{*}{$\begin{array}{l}\text { Erfragter Anwendungsbereich/ } \\
\text { Indikation }\end{array}$} & \multirow{2}{*}{$\begin{array}{l}\text { Indikation laut } \\
\text { BV-ÄLRD }\end{array}$} & \multicolumn{2}{|l|}{ Anzahl } & \multicolumn{3}{|c|}{ Bewertung } \\
\hline & & & $\begin{array}{l}\text { Mittelwert } \\
(n=71)\end{array}$ & $\begin{array}{l}\text { Standard- } \\
\text { abweichung } \\
(n=71)\end{array}$ & $\begin{array}{l}\text { Feste } \\
\text { Dosis }\end{array}$ & $\begin{array}{l}\text { Schwer- } \\
\text { wiegende } \\
\text { Komplikatio- } \\
\text { nen }\end{array}$ & $\begin{array}{l}\text { Vitale In- } \\
\text { dikation }\end{array}$ \\
\hline \multirow[t]{6}{*}{ Antiemetika } & MCP innerklinisch & \multirow{6}{*}{$\begin{array}{l}\text { Starke Übelkeit } \\
\text { und Erbrechen }\end{array}$} & 2,81 & 8,23 & \multirow[t]{2}{*}{ Ja } & \multirow[t]{2}{*}{$\mathrm{Ja}$} & \multirow[t]{6}{*}{ Nein } \\
\hline & MCP präklinisch & & 1,62 & 4,47 & & & \\
\hline & Ondansetron innerklinisch & & 14,38 & 35,06 & \multirow[t]{2}{*}{$\mathrm{Ja}$} & \multirow[t]{2}{*}{ Nein } & \\
\hline & Ondansetron präklinisch & & 1,51 & 5,50 & & & \\
\hline & Dimenhydrinat innerklinisch & & 6,38 & 11,63 & \multirow[t]{2}{*}{$\mathrm{Ja}$} & \multirow[t]{2}{*}{ Eher nein } & \\
\hline & Dimenhydrinat präklinisch & & 8,14 & 10,14 & & & \\
\hline \multirow[t]{2}{*}{ Furosemid } & Innerklinisch & \multirow[t]{2}{*}{ Lungenödem } & 4,65 & 7,87 & \multirow[t]{2}{*}{ Ja } & \multirow[t]{2}{*}{ Nein } & \multirow[t]{2}{*}{ Ja } \\
\hline & Präklinisch & & 6,06 & 9,45 & & & \\
\hline \multirow[t]{2}{*}{ Heparin } & Innerklinisch & \multirow[t]{2}{*}{ ACS } & 7,83 & 10,33 & \multirow[t]{2}{*}{$\mathrm{Ja}$} & \multirow[t]{2}{*}{ Nein } & \multirow[t]{2}{*}{$\mathrm{Ja}$} \\
\hline & Präklinisch & & 12,00 & 14,32 & & & \\
\hline ASS & Innerklinisch & ACS & 3,18 & 6,65 & $\mathrm{Ja}$ & Nein & $\mathrm{Ja}$ \\
\hline & Präklinisch & & 14,32 & 14,29 & & & \\
\hline Glyceroltrinitrat & Innerklinisch & ACS, Lungen- & 2,14 & 3,49 & $\mathrm{Ja}$ & $\mathrm{Ja}$ & $\mathrm{Ja}$ \\
\hline & Präklinisch & & 12,25 & 10,87 & & & \\
\hline Urapidil $^{b}$ & Innerklinisch & Keine Nennung & 2,25 & 3,48 & Nein & $\mathrm{Ja}$ & Eher ja \\
\hline & Präklinisch & & 5,83 & 7,73 & & & \\
\hline Nitrendipin & Innerklinisch & Hypertone Krise & 0,21 & 1,23 & $\mathrm{Ja}$ & & \\
\hline & Präklinisch & & 0,06 & 0,23 & & & \\
\hline Nifedipin $^{b}$ & Innerklinisch und präklinisch & Keine Nennung & 0,21 & 0,86 & $\mathrm{Ja}$ & & \\
\hline Metamizol & Innerklinisch & Antipyretika, & 29,92 & 42,45 & $\mathrm{Ja}$ & Eher nein & Nein \\
\hline & Präklinisch & Analgesie & 8,59 & 12,51 & & & \\
\hline Butylscopolamin & Innerklinisch & Koliken & 2,58 & 4,32 & $\mathrm{Ja}$ & Nein & Nein \\
\hline & Präklinisch & & 4,03 & 5,54 & & & \\
\hline Ketamin/ & Innerklinisch & Analgesie & 2,20 & 5,96 & Nein & $\mathrm{Ja}$ & Nein \\
\hline Esketamin & Präklinisch & & 3,51 & 5,23 & & & \\
\hline Opiate/Opioide & Innerklinisch & Analgesie bei & 59,65 & 72,75 & Nein & Eher nein & Eher nein \\
\hline & Präklinisch & ACS und Trauma & 10,72 & 16,18 & & & \\
\hline Ibuprofen & Innerklinisch & Antipyretika, & 2,62 & 7,61 & $\mathrm{Ja}$ & Nein & Nein \\
\hline & Präklinisch & Analgesie & 0,41 & 2,45 & & & \\
\hline Paracetamol & Innerklinisch & Antipyretika, & 4,31 & 9,29 & $\mathrm{Ja}$ & Nein & Nein \\
\hline & Präklinisch & Analgesie & 1,25 & 2,82 & & & \\
\hline Lidocain $^{\mathrm{e}}$ & Innerklinisch & Intraossäre & 8,30 & 12,52 & $\mathrm{Ja}$ & Nein & Nein \\
\hline & Präklinisch & Punktion & 0,28 & 0,81 & & & \\
\hline Glukose & Innerklinisch & Hypoglykämie & 1,32 & 2,33 & Eher ja & Nein & $\mathrm{Ja}$ \\
\hline & Präklinisch & & 7,38 & 6,67 & & & \\
\hline Naloxon & Innerklinisch & Opiatintoxikation & 1,00 & 1,93 & Nein & Nein & Ja \\
\hline & Präklinisch & & 0,20 & 0,67 & & & \\
\hline Tranexamsäure ${ }^{b}$ & Innerklinisch & Keine Nennung & 2,56 & 10,39 & $\mathrm{Ja}$ & Eher nein & Ja \\
\hline & Präklinisch & & 0,10 & 0,34 & & & \\
\hline $\begin{array}{l}\text { 'Zusätzlich zu den } 1 \\
\text { kolloidale Lösunger } \\
\text { Ärztlichen Leiter Re } \\
\text { 'Diese Medikamen } \\
\text { dieser Studie aber r } \\
\text { 'Ausgenommen Ac } \\
\text { 'Ausgenommen Di } \\
\text { 'Und andere Lokala }\end{array}$ & $\begin{array}{l}\text { ier dargestellten Medikamenten wer } \\
\text { als Medikamente für die Gabe durch } \\
\text { tungsdienst (BV-ÄLRD) empfohlen, v } \\
\text { e wurden nicht im Medikamentenkat } \\
\text { iterfasst } \\
\text { renalin } \\
\text { nenhydrinat } \\
\text { ästhetika }\end{array}$ & $\begin{array}{l}\text { vom Bundesverband } \\
\text { fallsanitäter genann } \\
\text { e in der Untersuchur } \\
\text { des Bundesverband }\end{array}$ & $\begin{array}{l}\text { der Ärztlicher } \\
\text { t. Die intramus } \\
\text { ng aber nicht € } \\
\text { ds der Ärztliche }\end{array}$ & $\begin{array}{l}\text { Leiter Rettungs } \\
\text { <uläre Adrenalin } \\
\text { fragt } \\
\text { n Leiter Rettung }\end{array}$ & $\begin{array}{l}\text { enst noch } \\
\text { be wird e } \\
\text { jenst aufg }\end{array}$ & $\begin{array}{l}\text { ristalloide Lösung } \\
\text { enfalls vom Bund, wurden im }\end{array}$ & $\begin{array}{l}\text { sowie } \\
\text { verband der } \\
\text { hmen }\end{array}$ \\
\hline
\end{tabular}


tengaben verbindlich vorgeben. Beispielhaft seien hier die sogenannten NUN-Algorithmen für Niedersachsen genannt, welche eine wesentliche Ausbildungsgrundlage für die in Niedersachsen ausgebildeten Notfallsanitäter darstellen. In vielen anderen Bundesländern hat sich hierfür dagegen der Begriff der Standardarbeitsanweisungen etabliert. Die vom BV-ÄLRD vorgeschlagene Auswahl an Medikamenten hat somit bereits breite Anerkennung gefunden und stellt derzeit den allgemein akzeptierten Ausgangspunkt für die Umsetzung des NotSanG dar.

Gegenstand der Untersuchung waren daher primär die vom BV-ÄLRD genannten Medikamente in der vom BV-ÄLRD hierfür genannten Indikation. Darüber hinaus wurde nach weiteren, aus Sicht der Autoren, relevanten Medikamenten und Indikationen gefragt. Hierbei fragten wir sowohl nach Anzahl der jeweiligen Anwendungen als auch nach einer Selbsteinschätzung der Auszubildenden, wie sicher sich diese in Indikationsstellung und Verabreichung der jeweiligen Medikamente waren. Den Ergebnissen wurde eine Bewertung der einzelnen Substanzen bezüglich des Nutzens und des Vorhandenseins schwerwiegender Risiken gegenübergestellt. Ziel der Untersuchung war die Ermittlung, welche Expertise bzw. Anwendungssicherheit Notfallsanitäter im Rahmen ihrer Ausbildung bisher erlangen.

\section{Methodik}

Die Datenerhebung wurde von Juni 2017 bis August 2017 in drei niedersächsischen Rettungsdienstschulen durchgeführt. Die Erhebung erfolgte jeweils im Rahmen eines Termins von circa $3 \mathrm{~h}$ in den teilnehmenden Rettungsdienstschulen mit den dort beschulten Auszubildenden. Es wurden 71 Auszubildende zum Notfallsanitäter befragt, die die Ausbildung in Vollzeit absolvierten und in 20 verschiedenen Rettungsdienstbereichen im Land Niedersachsen beschäftigt waren. Die befragten Auszubildenden waren damit die ersten in Niedersachsen in Vollzeit ausgebildeten Notfallsanitäter. Alle Auszubildenden befanden sich zum Zeitpunkt ihrer Befragung kurz vor Abschluss ihrer Ausbildung, waren volljährig und hatten bereits alle Klinikpraktika und Rettungswachen- praktika absolviert. Die Auszubildenden wurden gebeten, einen Fragebogen mit 261 Fragen zu ihrer Ausbildung und fünf demografischen Fragen anonym zu beantworten. Dabei wurde nach der Anzahl der Anwendungen verschiedener Medikamente gefragt. Die Antworten wurden in eine Datenbank übertragen und es erfolgte eine deskriptive statistische Auswertung mit SOFA (Paton-Simpson and Associates Ltd, USA; • Abb. 1). Ein Teil der Ergebnisse dieser Untersuchung wurde bereits veröffentlicht [4].

Durch gezielte Fragestellungen erfolgte eine Unterteilung in einzelne relevante Ausbildungsbereiche, deren Rahmenbedingungen und Ausbildungsmöglichkeiten sich deutlich voneinander unterscheiden. In der Regel wurde für jedes Medikament jeweils nach der Anzahl innerklinisch und präklinisch durchgeführter Anwendungen gefragt. Die Antworten zur Anzahl der Medikamentenapplikationen sollten von den Auszubildenden, soweit möglich, aus ihrem Praktikumsheft entnommen werden, wo dies nicht möglich war sollten die Auszubildenden die Frage nach bestem Wissen beantworten und ggf. schätzen. Es wurden jeweils Mittelwert und Standardabweichung berechnet.

Die Auszubildenden wurden außerdem zu allen Medikamenten nach einer Selbsteinschätzung befragt. Dabei sollten die Auszubildenden ihre eigene Handlungskompetenz bezüglich der Anwendung der verschiedenen Notfallmedikamente im Gesamtzusammenhang eines Notfalleinsatzes in Schulnoten einschätzen. Dabei entsprach die Note 1 "sehr gut", wohingegen die Note 6 als schlechteste Note "ungenügend" und somit gravierenden Defiziten entsprach. Für die Notenverteilung wurde der Median berechnet, für das Verhältnis zwischen Anzahl durchgeführter Medikamentengaben und Selbsteinschätzung der Auszubildenden wurde der Korrelationskoeffizient nach Spearman berechnet.

Die Teilnahme an der Untersuchung war freiwillig und wurde durch die Rettungsdienstschulen, an denen die Befragung stattfand, freundlicherweise unterstützt. Mit der Teilnahme erklärten sich die Auszubildenden mit einer Veröffentlichung der Daten einverstanden.
Die vom BV-ÄLRD genannten Medikamente wurden von den Autoren jeweils unter den Aspekten Vorhandensein einer festen Dosierungsangabe, Vorhandensein potenzieller schwerwiegender Komplikationen bei gegebener Indikation und nach dem Vorhandensein einer vitalen Indikation bewertet.

\section{Ergebnisse}

Insgesamt nahmen 71 Auszubildende zum Notfallsanitäter an der Befragung teil. Die Auszubildenden waren im Mittel 22,8 Jahre alt (SD 2,1), der jüngste Teilnehmer war 20 Jahre alt, wohingegen der älteste zum Zeitpunkt der Befragung 31 Jahre alt war. Es nahmen 48 männliche und 23 weibliche Auszubildende an der Befragung teil.

- Tab. 1 zeigt die Anzahl der Verabreichungen einzelner Medikamente unter besonderer Berücksichtigung des vom BVÄLRD vorgeschlagenen Medikamentenkatalogs. Die Ergebnisse sind jeweils als Mittelwert und Standardabweichung dargestellt.

- Tab. 2 zeigt die Selbsteinschätzung der Auszubildenden bezüglich der verschiedenen Medikamente auf einer Schulnotenskala von 1 bis 6 . Dargestellt werden jeweils der Median der Notenverteilung sowie die Spearman-Korrelation zwischen Anzahl von Medikamentenapplikationen und Selbsteinschätzung der Studierenden für die einzelnen Medikamente.

\section{Diskussion}

Die vom BV-ÄLRD für die Anwendung durch Notfallsanitäter vorgeschlagenen Medikamente sind in vielerlei Hinsicht sehr heterogen. Ein Teil der Medikamente ist so unmittelbar lebensrettend, dass bei entsprechender Indikation Bedenken bezüglich Nebenwirkungen, Wechselwirkungen und vermeintlicher Kontraindikationen stark in den Hintergrund treten. Einige Medikamente haben dagegen wenig bis keinen Einfluss auf das Überleben der Patienten, sodass eine Gabe nur unter strikter Beachtung von Nebenund Wechselwirkungen gerechtfertigt erscheint. Ein Teil der vorgeschlagenen Medikamente hat wenig bis keine für den Rettungsdienst relevanten Wechsel- und Nebenwirkungen, bei anderen ist für die 
Tab. 2 Selbsteinschätzung der Auszubildenden in Schulnoten von 1 bis 6 zur Sicherheit in der Indikationsstellung und Verabreichung von Medikamenten, unter besonderer Berücksichtigung des vom Bundesverband der Ärztlichen Leiter Rettungsdienst veröffentlichten Medikamentenkatalogs [ 1 ], und Korrelation zwischen Anzahl durchgeführter Maßnahmen und Selbsteinschätzung

Medikament/
Fragestellung

Selbsteinschätzung Note 1-6

\begin{tabular}{|l|l|l|l|l|} 
& Median & $\begin{array}{l}\text { Korrelation } \\
\text { r }\end{array}$ & & \multicolumn{2}{|l|}{ Notenverteil } \\
\cline { 4 - 5 } & & & $\mathbf{1}$ & \\
\cline { 4 - 5 } & & $\begin{array}{l}n \\
(\%)\end{array}$ & \\
\hline Adrenalin intravenös bei & 4 & 0,18 bzw. & 0 &
\end{tabular}

Anaphylaxie oder Brady

kardie

\begin{tabular}{|l|l|l|}
\hline Adrenalin inhalativ & 2 & 0,06
\end{tabular}

\begin{tabular}{l|l} 
Noradrenalin $^{\mathrm{a}}$ & 4 \\
\hline
\end{tabular}

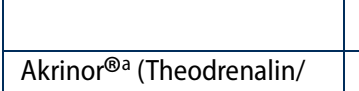

Cafedrin)

Amiodaron bei Reanimati-

on

Amiodaron bei tachykar- 5

den Herzrhythmusstörun-

gen

\begin{tabular}{l|l|l}
\hline Atropin & 3 & -0,
\end{tabular}

Benzodiazepine $^{\mathrm{b}}$

Beta-2-Mimetika intrave-

nös und inhalativ ${ }^{b, c}$

$4 \quad-0,03$

4

\begin{tabular}{l|l}
$0,033^{k}$ & $(0)$ \\
\hline
\end{tabular}

\begin{tabular}{l|l}
7 & 32 \\
$(9,86)$ & 145,0
\end{tabular}

\begin{tabular}{l|l}
\hline$(9,86)$ & 145 \\
\hline 0 & 4
\end{tabular}

\begin{tabular}{l|l}
\hline 2 & \\
\hline & $\%)$
\end{tabular}

\begin{tabular}{|l|l|}
\hline 3 & 4 \\
\hline$n$ & $\%)$
\end{tabular}

\begin{tabular}{|l|l|l|}
\hline $\mathbf{4}$ & $\mathbf{5}$ & 6 \\
\hline$n$ & $n$ & \\
$(\%)$ & $(\%)$ & $(\%)$ \\
\hline
\end{tabular}

\begin{tabular}{|l|l|l|l|l}
\hline 7 & 21 & 22 & 17 & 4
\end{tabular}

$\%)$

\begin{tabular}{|l|l|}
\hline & \\
\hline
\end{tabular}

\begin{tabular}{|l|l|l|}
\hline Ipratropiumbromid & 3 & $-0,5$ \\
\hline Kortikosteroide $^{\mathrm{b}}$ & 3 & $-0,2$ \\
\hline Histaminblocker & & $0, \mathrm{~d}$ \\
\hline
\end{tabular}

\begin{tabular}{|l|l|l|l|}
\hline Histaminblocker $^{\mathrm{b}, \mathrm{d}}$ & 4 & $\begin{array}{l}-0,31^{\mathrm{p}, \mathrm{s}} \\
-0,21^{\mathrm{k}}\end{array}$ & 3 \\
\hline Antiemetika $^{\mathrm{b}}$ & 2 & $/$ & 4
\end{tabular}

\begin{tabular}{|l|}
\hline Metoclopramid \\
\hline
\end{tabular}

\begin{tabular}{|l|l|l|}
\hline Ondansetron & 4 & -0, \\
\hline
\end{tabular}

Dimenhydrinat

Furosemid

3

\begin{tabular}{|c|c|c|c|c|c|c|c|c|c|}
\hline & & $-0,18^{k}$ & $(5,63)$ & $(35,21)$ & $(40,85)$ & $(15,49)$ & $(1,41)$ & $(1,41)$ & \\
\hline Heparin & 3 & $\begin{array}{l}-0,08^{p} \\
-0,01^{k}\end{array}$ & $\begin{array}{l}3 \\
(4,23)\end{array}$ & $\begin{array}{l}30 \\
(42,25)\end{array}$ & $\begin{array}{l}29 \\
(40,85)\end{array}$ & $\begin{array}{l}6 \\
(8,45)\end{array}$ & $\begin{array}{l}2 \\
(2,82)\end{array}$ & $\begin{array}{l}1 \\
(1,41)\end{array}$ & 71 \\
\hline Acetylsalicylsäure & 2 & $\begin{array}{l}-0,02^{p} \\
0,24^{k}\end{array}$ & $\begin{array}{l}8 \\
(11,27)\end{array}$ & $\begin{array}{l}40 \\
(56,34)\end{array}$ & $\begin{array}{l}19 \\
(26,76)\end{array}$ & $\begin{array}{l}4 \\
(5,63)\end{array}$ & $\begin{array}{l}0 \\
(0)\end{array}$ & $\begin{array}{l}0 \\
(0)\end{array}$ & 71 \\
\hline $\begin{array}{l}\text { Glyceroltrinitrat (Nitro- } \\
\text { spray) }\end{array}$ & 2 & $\begin{array}{l}0,02^{p} \\
0,13^{k}\end{array}$ & $\begin{array}{l}9 \\
(12,68)\end{array}$ & $\begin{array}{l}42 \\
(59,15)\end{array}$ & $\begin{array}{l}16 \\
(22,54)\end{array}$ & $\begin{array}{l}3 \\
(4,23)\end{array}$ & $\begin{array}{l}1 \\
(1,41)\end{array}$ & $\begin{array}{l}0 \\
(0)\end{array}$ & 71 \\
\hline Urapidil $^{\mathrm{a}}$ & 3 & $\begin{array}{l}-0,23^{p} \\
0,05^{k}\end{array}$ & $\begin{array}{l}1 \\
(1,41)\end{array}$ & $\begin{array}{l}24 \\
(33,80)\end{array}$ & $\begin{array}{l}34 \\
(47,89)\end{array}$ & $\begin{array}{l}9 \\
(12,68)\end{array}$ & $\begin{array}{l}3 \\
(4,23)\end{array}$ & $\begin{array}{l}0 \\
(0)\end{array}$ & 71 \\
\hline Nitrendipin & 6 & $\begin{array}{l}0,40^{\mathrm{p}, \mathrm{s}} \\
0,43^{\mathrm{k}, \mathrm{s}}\end{array}$ & $\begin{array}{l}0 \\
(0)\end{array}$ & $\begin{array}{l}1 \\
(1,41)\end{array}$ & $\begin{array}{l}2 \\
(2,82)\end{array}$ & $\begin{array}{l}5 \\
(7,04)\end{array}$ & $\begin{array}{l}13 \\
(18,31)\end{array}$ & $\begin{array}{l}50 \\
(70,42)\end{array}$ & 71 \\
\hline
\end{tabular}




\begin{tabular}{|c|c|c|c|c|c|c|c|c|c|}
\hline \multirow{4}{*}{$\begin{array}{l}\text { Medikament/ } \\
\text { Fragestellung }\end{array}$} & \multicolumn{8}{|c|}{ Selbsteinschätzung Note 1-6 } & \multirow[t]{4}{*}{$n$ gesamt } \\
\hline & \multirow[t]{3}{*}{ Median } & \multirow{3}{*}{$\begin{array}{l}\text { Korrelation } \\
r_{\mathrm{s}}\end{array}$} & \multicolumn{6}{|c|}{ Notenverteilung } & \\
\hline & & & 1 & 2 & 3 & 4 & 5 & 6 & \\
\hline & & & $\begin{array}{l}n \\
\text { (\%) }\end{array}$ & $\begin{array}{l}n \\
\text { (\%) }\end{array}$ & $\begin{array}{l}n \\
\text { (\%) }\end{array}$ & $\begin{array}{l}n \\
\text { (\%) }\end{array}$ & $\begin{array}{l}n \\
\text { (\%) }\end{array}$ & $\begin{array}{l}n \\
\text { (\%) }\end{array}$ & \\
\hline Metamizol & 2 & $\begin{array}{l}-0,22^{\mathrm{p}} \\
-0,32^{\mathrm{k}, \mathrm{s}}\end{array}$ & $\begin{array}{l}3 \\
(4,29)\end{array}$ & $\begin{array}{l}35 \\
(50,00)\end{array}$ & $\begin{array}{l}26 \\
(37,14)\end{array}$ & $\begin{array}{l}4 \\
(5,71)\end{array}$ & $\begin{array}{l}2 \\
(2,86)\end{array}$ & $\begin{array}{l}0 \\
(0)\end{array}$ & $70^{e}$ \\
\hline Butylscopolamin & 3 & $\begin{array}{l}-0,23^{p} \\
0,01^{k}\end{array}$ & $\begin{array}{l}3 \\
(4,23)\end{array}$ & $\begin{array}{l}24 \\
(33,80)\end{array}$ & $\begin{array}{l}31 \\
(43,66)\end{array}$ & $\begin{array}{l}11 \\
(15,49)\end{array}$ & $\begin{array}{l}1 \\
(1,41)\end{array}$ & $\begin{array}{l}1 \\
(1,41)\end{array}$ & 71 \\
\hline Ketamin/Esketamin & 3 & $\begin{array}{l}-0,25^{p} \\
0,21^{k}\end{array}$ & $\begin{array}{l}1 \\
(1,42)\end{array}$ & $\begin{array}{l}12 \\
(17,14)\end{array}$ & $\begin{array}{l}23 \\
(32,86)\end{array}$ & $\begin{array}{l}28 \\
(40,00)\end{array}$ & $\begin{array}{l}5 \\
(7,14)\end{array}$ & $\begin{array}{l}1 \\
(1,42)\end{array}$ & $70^{\mathrm{e}}$ \\
\hline Opiate/Opioide & 3 & $\begin{array}{l}-0,12^{p} \\
-0,24^{k}\end{array}$ & $\begin{array}{l}3 \\
(4,23)\end{array}$ & $\begin{array}{l}14 \\
(19,72)\end{array}$ & $\begin{array}{l}37 \\
(52,11)\end{array}$ & $\begin{array}{l}9 \\
(12,68)\end{array}$ & $\begin{array}{l}8 \\
(11,27)\end{array}$ & $\begin{array}{l}0 \\
(0)\end{array}$ & 71 \\
\hline Ibuprofen & 4 & $\begin{array}{l}0,24^{\mathrm{p}} \\
0,09^{\mathrm{k}}\end{array}$ & $\begin{array}{l}3 \\
(4,23)\end{array}$ & $\begin{array}{l}16 \\
(22,54)\end{array}$ & $\begin{array}{l}16 \\
(22,54)\end{array}$ & $\begin{array}{l}9 \\
(12,68)\end{array}$ & $\begin{array}{l}15 \\
(21,13)\end{array}$ & $\begin{array}{l}12 \\
(16,90)\end{array}$ & 71 \\
\hline Paracetamol & 3 & $\begin{array}{l}-0,03^{p} \\
-0,05^{k}\end{array}$ & $\begin{array}{l}0 \\
(0)\end{array}$ & $\begin{array}{l}19 \\
(26,76)\end{array}$ & $\begin{array}{l}23 \\
(32,39)\end{array}$ & $\begin{array}{l}17 \\
(23,94)\end{array}$ & $\begin{array}{l}10 \\
(14,08)\end{array}$ & $\begin{array}{l}2 \\
(2,82)\end{array}$ & 71 \\
\hline $\begin{array}{l}\text { Lidocain oder andere Lo- } \\
\text { kalanästhetika }\end{array}$ & 4 & $\begin{array}{l}0,28^{p} \\
-0,30^{k, s}\end{array}$ & $\begin{array}{l}0 \\
(0)\end{array}$ & $\begin{array}{l}3 \\
(4,23)\end{array}$ & $\begin{array}{l}12 \\
(16,90)\end{array}$ & $\begin{array}{l}30 \\
(42,25)\end{array}$ & $\begin{array}{l}12 \\
(16,90)\end{array}$ & $\begin{array}{l}14 \\
(19,72)\end{array}$ & 71 \\
\hline Glukose & 2 & $\begin{array}{l}-0,09^{p} \\
0,21^{k}\end{array}$ & $\begin{array}{l}16 \\
(22,54)\end{array}$ & $\begin{array}{l}41 \\
(57,75)\end{array}$ & $\begin{array}{l}12 \\
(16,90)\end{array}$ & $\begin{array}{l}2 \\
(2,82)\end{array}$ & $\begin{array}{l}0 \\
(0)\end{array}$ & $\begin{array}{l}0 \\
(0)\end{array}$ & 71 \\
\hline Naloxon & 4 & $\begin{array}{l}0,29^{p} \\
-0,12^{k}\end{array}$ & $\begin{array}{l}1 \\
(1,41)\end{array}$ & $\begin{array}{l}7 \\
(9,86)\end{array}$ & $\begin{array}{l}16 \\
(22,54)\end{array}$ & $\begin{array}{l}17 \\
(23,94)\end{array}$ & $\begin{array}{l}18 \\
(25,35)\end{array}$ & $\begin{array}{l}12 \\
(16,90)\end{array}$ & 71 \\
\hline Tranexamsäure $^{a}$ & 6 & $\begin{array}{l}0,30^{\mathrm{p}, \mathrm{s}} \\
0,21^{\mathrm{k}}\end{array}$ & $\begin{array}{l}1 \\
(1,41)\end{array}$ & $\begin{array}{l}1 \\
(1,41)\end{array}$ & $\begin{array}{l}4 \\
(5,63)\end{array}$ & $\begin{array}{l}8 \\
(11,27)\end{array}$ & $\begin{array}{l}15 \\
(21,13)\end{array}$ & $\begin{array}{l}42 \\
(59,15)\end{array}$ & 71 \\
\hline \multicolumn{10}{|c|}{ 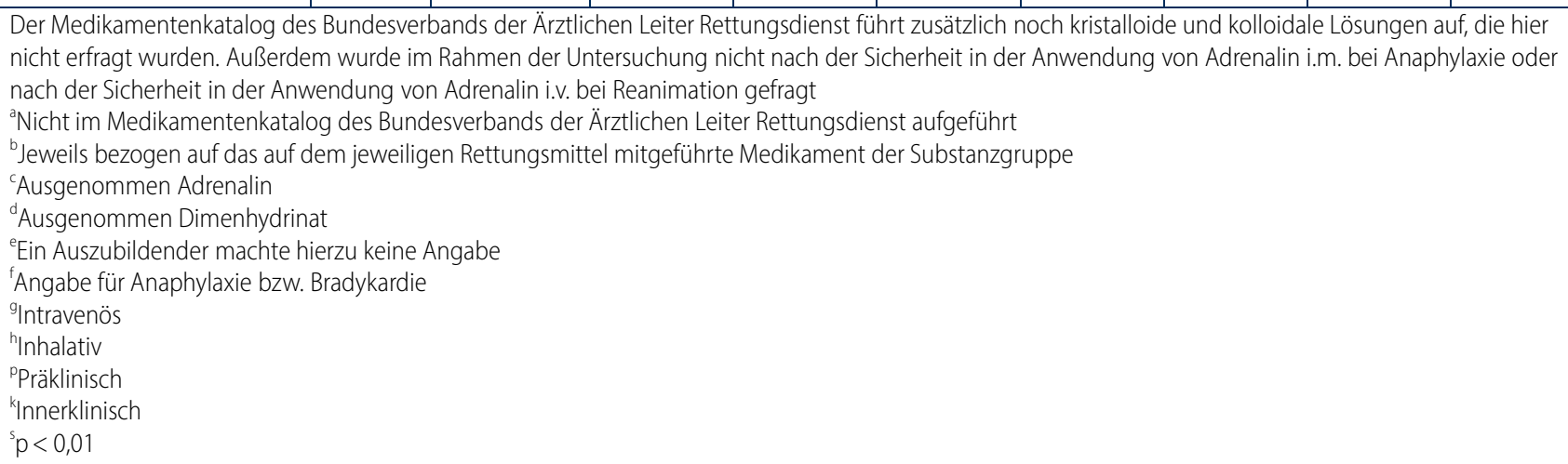 } \\
\hline
\end{tabular}

korrekte Anwendung eine hohe klinische Erfahrung notwendig. So ist die Gabe von Furosemid bei kardialem Lungenödem deutlich anders zu bewerten als die Gabe von MCP bei Übelkeit. Im Folgenden sollen daher die einzelnen Medikamente nach Substanzgruppe bzw. Indikation geordnet besprochen werden.

Kardiovaskulär wirksame Medikamente und Antiarrhythmika

Adrenalin wurde 9,76 (13,31) Mal von den Auszubildenden im Rahmen einer Reanimation eingesetzt. Die korrekte Dosierung und Indikationsstellung sind hier einfach durch Algorithmen abbildbar und die kardiopulmonale Reanimation ein vergleichsweise häufiges Notfallbild. Die Verabreichung von Adrenalin i.v. im Rahmen von Bradykardien oder Anaphylaxie wurde dagegen, mit 0,28 (0,70) bzw. 2,22 (6,67) Anwendungen, selten durchgeführt. Die Wahl der korrekten Dosierung wird zwar ebenfalls über Algorithmen vorgegeben, in der klinischen Realität erfordert die Dosisfindung jedoch Erfahrung, um die Substanz zielgerichtet einsetzen zu können. Aufgrund der seltenen Anwendung ist davon auszugehen, dass diese Erfahrung bei den befragten Auszubildenden mehrheitlich nicht besteht, was sich auch im Ergeb- nis der Selbsteinschätzung mit der Note 4 widerspiegelt. Im Gegensatz dazu konnten die Auszubildenden mit dem klinisch häufiger eingesetzten Noradrenalin mit 9,4 $(16,07)$ Anwendungen mehr Erfahrungen sammeln. Die Auszubildenden schätzten sich selbst allerdings mit einer Note von 4 hier ebenfalls zurückhaltend ein. Die Gabe von Adrenalin i.m. im Rahmen einer anaphylaktischen Reaktion war nicht Bestandteil dieser Befragung. Aufgrund der vergleichsweise einfachen Dosierung bei intramuskulärer Verabreichung ist die Komplexität der Maßnahme aus Sicht der Autoren als eher niedrig zu bewerten. Dementsprechend sollte eine ausreichende Exper- 
tise mit wenig Erfahrung bereits erworben werden können und daher mit Abschluss der Ausbildung vermutlich bestehen.

Die Gabe von Akrinor ${ }^{\circledR}$ ist vergleichsweise einfacher und daher möglicherweise ebenfalls eine gute Alternative zur Behandlung einer Hypotonie für im Umgang mit Katecholaminen wenig erfahrene Anwender. Die Auszubildenden hatten hier mit 10,99 $(15,25)$ Anwendungen ebenfalls häufig die Chance, diese Substanz klinisch einzusetzen. Trotzdem war auch hier die Selbsteinschätzung der Auszubildenden mit Note 4 im Median eher schlecht.

Die Auszubildenden hatten mit 2,46 $(3,46)$ Anwendungen in der Regel die Gelegenheit, prähospital im Rahmen einer Reanimation Amiodaron zu verabreichen. Dosierung und Indikation sind einfach und die Selbsteinschätzung mit Note 2 erwartbar gut. Im Rahmen von tachykarden Rhythmusstörungen bei stabilen Patienten hatten die Auszubildenden dagegen weder klinisch mit 0,72 $(2,05)$ noch präklinisch mit 0,55 $(2,43)$ Anwendungen, die Gelegenheit, häufig Erfahrungen im Umgang mit dieser Substanz zu machen. Die Therapie tachykarder Herzrhythmusstörungen ist nicht trivial. Die Selbsteinschätzung der Auszubildenden war mit Note 5 erwartbar schlecht.

Atropin wurde mit 10,75 $(11,91)$ innerklinischen und 1,18 $(1,97)$ präklinischen Anwendungen von den Auszubildenden häufig verabreicht. Die meisten Auszubildenden hatten somit Gelegenheit, Indikationsstellung und Anwendung der Substanz kennenzulernen. Für die Verabreichung der Substanz besteht eine feste Dosierung von 0,5 mg, welche in der Regel den Inhalt einer Ampulle darstellt, was eine einfache Dosierung ermöglicht. Es muss daher davon ausgegangen werden, dass die Auszubildenden hier eine gewisse Expertise erwerben konnten. Die Selbsteinschätzung spiegelt dies mit der Note 3 wider.

\section{Bronchodilatatoren und Kortikosteroide}

Inhalative Beta-2-Mimetika wurden von den Auszubildenden sowohl klinisch mit $6,07(11,06)$ Anwendungen als auch präklinisch mit 8,89 $(8,06)$ Anwendungen häufig verabreicht. Inhalatives Adrenalin da- gegen deutlich seltener mit $0,73(1,03)$ Anwendungen. Die Dosierung dieser Substanzen ist einfach und es bestand die Gelegenheit, die Substanzen im Rahmen der Ausbildung kennenzulernen. Die Selbsteinschätzung der Auszubildenden ist für beide Substanzen mit Note 2 dementsprechend gut. Intravenöse Beta-2-Mimetika wurden dagegen von den Auszubildenden selten verabreicht, sodass hier keine ausreichende Erfahrung besteht. Die Anwendung durch Notfallsanitäter muss, aufgrund der hämodynamischen Nebenwirkungen, auch kritisch betrachtet werden.

Ipratropiumbromid wurde ebenfalls sowohl klinisch mit 2,20 $(4,49)$ Anwendungen als auch präklinisch mit 3,70 $(5,91)$ Anwendungen häufig verabreicht, wenn auch insgesamt seltener als inhalative Beta-2Mimetika. Auch hier besteht eine Dosierung, die einer Ampullengröße entspricht. Die Selbsteinschätzung war mit Note 3 geringfügig schlechter als für Betamimetika.

Kortikosteroide wurden klinisch mit $3,10(8,24)$ Anwendungen wie präklinisch mit 3,79 $(5,32)$ Anwendungen gleichermaßen häufig verabreicht. Die Selbsteinschätzung lag im Median bei 3 und es kann dementsprechend von einer ausreichenden Erfahrung im Umgang mit diesen Medikamenten ausgegangen werden.

\section{Antihistaminika}

Insbesondere innerklinisch wurden Antihistaminika häufig verabreicht mit 6,62 $(29,37)$ Anwendungen pro Auszubildenden. Präklinisch wurden Antihistaminika 1,82 $(4,34)$ Mal von Auszubildenden verabreicht. Die Selbsteinschätzung der Auszubildenden war mit Note 4 im Median dagegen aber eher schlecht. Da Dosierung und Indikationsstellung vergleichsweise einfach sind, sollte die Anwendung von diesen Substanzen im Rahmen der Ausbildung erlernbar sein.

\section{Antiemetika}

Übelkeit und Erbrechen, wie sie auf einem Transport in einem Rettungsmittel häufig auftreten, sind nicht nur unangenehm für den Patienten, sondern erhöhen auch Stress und Sauerstoffverbrauch und können das Outcome des Patienten möglicherweise negativ beeinflussen. Auch wenn es sich bei der Gabe von Antiemetika nicht um eine unmittelbar lebensrettende Therapie handelt, scheint die Applikation dieser Medikamente durch Notfallsanitäter daher grundsätzlich sinnvoll. Das innerklinisch am häufigsten verabreichte Antiemetikum war Ondansetron mit 14,38 $(35,06)$ Anwendungen. Präklinisch wurde Ondansetron dafür vergleichsweise selten verabreicht mit 1,51 (5,50) Anwendungen. Präklinisch wurde von den Notfallsanitätern vor allem Dimenhydrinat verabreicht mit 8,14 $(10,14)$ Anwendungen. Innerklinisch wurde Dimenhydrinat $6,38(11,63)$ Mal von den Notfallsanitätern verabreicht. MCP wurde dagegen mit 2,81 $(8,23)$ innerklinischen und 1,62 $(4,47)$ präklinischen Anwendungen am seltensten verabreicht. Die Selbsteinschätzung war mit Note 2 für Dimenhydrinat deutlich besser als für MCP mit 5 oder Ondansetron mit 4. Dies ist vor dem Hintergrund der häufigen Anwendung von Ondansetron und der damit verbundenen mit Abstand größten Erfahrung im Umgang mit dieser Substanz sowie des geringen Nebenwirkungsprofils nicht nachvollziehbar. Möglicherweise sind die weite Verbreitung von Dimenhydrinat im Rettungsdienst und die höhere Erfahrung im präklinischen Bereich hier ursächlich für die bessere Selbsteinschätzung im Einsatz dieser Substanz. Für Dimenhydrinat und Ondansetron kann eine entsprechende Erfahrung im Umgang mit diesen Substanzen angenommen werden. Für MCP bestand hier eher keine ausreichende Erfahrung und das Medikament erscheint bei entsprechenden Alternativen eher ungeeignet. Dies gilt insbesondere vor dem Hintergrund der 2014 erfolgten Indikationseinschränkung bei negativem NutzenRisiko-Verhältnis durch das BfArM. Ondansetron stellt möglicherweise die günstigste Substanz für den Einsatz durch Notfallsanitäter dar, hat den Sprung in die Präklinik allerdings bisher immer noch nicht geschafft.

\section{Furosemid, Glyceroltrinitrat und Antihypertensiva}

Furosemid wurde von den Auszubildenden innerklinisch 4,65 $(7,87)$ und präklinisch 6,06 $(9,45)$ Mal verabreicht. Die Auszubildenden schätzten sich bezogen auf die Sicherheit der Indikationsstellung und 
Applikation selbst im Median mit Note 3 ein. Nach Einschätzung der Autoren kann damit davon ausgegangen werden, dass im Rahmen der Ausbildung ausreichende Erfahrungen gesammelt werden, um diese Substanz präklinisch sinnvoll einsetzen zu können.

Glyceroltrinitrat wurde insbesondere präklinisch von den Auszubildenden häufig eingesetzt. Hier hatten die Auszubildenden die Gelegenheit, die Substanz $12,25(10,87)$ Mal anzuwenden. Die Selbsteinschätzung der Auszubildenden lag im Median bei Note 2. Die Erfahrung aus dem Einsatz von Glyceroltrinitrat durch Rettungsassistenten im Rahmen der sogenannten "Notkompetenz" [5] zeigt ebenfalls, dass der Einsatz durch Rettungsdienstpersonal erlernbar ist und in der Regel beherrscht wird.

Urapidil ist ein im Notarztdienst weit verbreitetes Antihypertensivum. Der blutdrucksenkende Effekt ist in der Regel gut steuerbar. Vom BV-ÄLRD wurde die Substanz allerdings nicht für den Einsatz durch Notfallsanitäter vorgeschlagen. Die Substanz muss zwar titrierend verabreicht werden, da es bei Überdosierung schnell zu einer relevanten Hypotonie kommen kann, allerdings scheint diese Erfahrung grundsätzlich im Rahmen der Ausbildung erwerbbar. Im Rahmen dieser Untersuchung gaben die Auszubildenden an, die Substanz 2,25 $(3,48)$ Mal innerklinisch und $5,83(7,73)$ Mal präklinisch angewendet zu haben. Es besteht also eine gewisse Erfahrung im Umgang mit dieser Substanz. Dies zeigt auch die Selbsteinschätzung der Auszubildenden mit einer Note von 3.

Das vom BV-ÄLRD für die Gabe durch Notfallsanitäter vorgeschlagene Nitrendipin wurde dagegen nicht häufig eingesetzt - weder klinisch mit $0,21(1,23)$ noch präklinisch mit 0,06 $(0,23)$ Anwendungen. Die hier befragten Auszubildenden hatten daher keine Möglichkeit, im Umgang mit Nitrendipin Erfahrungen zu sammeln. Die Selbsteinschätzung spiegelt dies mit Note 6 deutlich wider. Hinzu kommt, dass die Substanz aufgrund der schlechten Steuerbarkeit als eher ungeeignet für den Einsatz im Rettungsdienst erscheint. Aufgrund des innerklinisch häufigen Einsatzes von Nifedipin befragten wir die Auszubildenden auch zu dieser ebenfalls nicht im Katalog des BV-ÄLRD vorgeschlagenen
Substanz. Die Auszubildenden hatten hier allerdings gleichfalls kaum Erfahrungen sammeln können.

\section{Antikoagulanzien und Thrombozy- tenaggregationshemmer}

Aufgrund der hohen Inzidenz des akuten Koronarsyndroms im Rettungsdienst hatten die Auszubildenden insbesondere präklinisch häufig die Gelegenheit, Acetylsalicylsäure (ASS) und Heparin zu verabreichen. ASS wurde dabei 14,32 $(14,29)$ und Heparin 12,00 (14,32) Mal von den Auszubildenden verabreicht. Die Auszubildenden fühlten sich in der Applikation dieser Substanzen sicher und gaben im Rahmen der Selbsteinschätzung im Median eine Note von 2 für ASS und 3 für Heparin an. Das große Spektrum der Differenzialdiagnosen akuter Brustschmerzen beinhaltet auch Erkrankungen, bei denen die Applikation dieser Substanzen kontraindiziert ist und die sich im Rettungs- und Notarztdienst oft nicht genauer abklären lassen. Es handelt es sich daher oft um eine individuelle klinische Abwägung, die sich schlecht in Rahmen einer algorithmenbasierten Anwendung abbilden lässt. Insgesamt scheint das Nutzen-Risiko Verhältnis jedoch für die frühe Applikation dieser Substanzen durch Notfallsanitäter zu sprechen.

\section{Analgetika}

Die Gabe von Analgetika durch Rettungsdienstpersonal wurde bereits 1992 in der Stellungnahme der Bundesärztekammer zur "Notkompetenz" für Rettungsassistenten erwähnt [5]. Dies führte allerdings in den seltensten Fällen tatsächlich zu einer Freigabe von Analgetika durch den verantwortlichen Ärztlichen Leiter Rettungsdienst. Mit der Einführung des Notfallsanitäters wird dies erneut diskutiert. Auch gegenwärtig ist die Wahl eines geeigneten Analgetikums für den Einsatz durch Rettungsdienstpersonal schwierig und teilweise durch gesetzliche Normen eingeschränkt.

Die größte Erfahrung konnten die Auszubildenden mit Opiaten bzw. Opioiden sammeln, dies insbesondere im innerklinischen Bereich mit 59,65 (72,75) Anwendungen. Aber auch präklinisch bestand mit 10,72 $(16,18)$ Anwendungen die Gelegenheit, Erfahrungen mit dieser Substanzgruppe zu sammeln. Die Selbsteinschätzung der Auszubildenden ergab im Median die Note 3. Die Dosisfindung setzt zwar eine Titration voraus, diese scheint aber im Rahmen der häufigen Anwendung erlernbar zu sein. Insgesamt muss eine ausreichende Expertise angenommen werden. Vor diesem Hintergrund scheint die Freigabe von Opiaten bzw. Opioiden aus medizinischer Sicht gerechtfertigt. Darüber hinaus existieren bereits seit 2007 Daten, die zeigen, dass die Gabe von Opiaten bzw. Opioiden durch Rettungsdienstpersonal sicher und effektiv erfolgen kann [6, 7].

Für die Verabreichung von Ketamin existieren ähnliche Studien [8, 9] und die Substanz wird vom BV-ÄLRD explizit für die Gabe durch Notfallsanitäter empfohlen. In dieser Untersuchung wurde Ketamin jedoch im Vergleich deutlich seltener eingesetzt. Klinisch wendeten die Auszubildenden Ketamin 2,20 (5,96) Mal an, präklinisch 3,51 $(5,23)$ Mal. Die Selbsteinschätzung der Auszubildenden lag im Median bei Note 3. Vor dem Hintergrund eines fließenden Übergangs zwischen Analgesie, Analgosedierung und Narkose, insbesondere bei Kombination mit einem Benzodiazepin, mit allen hiermit verbundenen Implikationen, des beträchtlichen pharmakologischen Unterschieds zwischen Ketamin und Esketamin, der deutlich selteneren Anwendung der Substanz verglichen mit Opiatanalgetika und des Fehlens eines Antidots scheint diese Substanz insgesamt für die Gabe durch Notfallsanitäter schlechter geeignet. Gerade in Bezug auf die Analgesie muss angemerkt werden, dass es sich - bei allen guten Gründen, die für eine frühzeitige und bedarfsgerechte Schmerztherapie sprechen - in der Regel eher nicht um eine vitale Indikation handelt. Ein größtmögliches $M a ß$ an Patientensicherheit ist hier, aus Sicht der Autoren, höher zu werten als eine frühere Gabe.

Metamizol wurde ebenfalls sehr häufig durch die Auszubildenden verabreicht. Innerklinisch verabreichten die Auszubildenden Metamizol im Schnitt 29,92 $(42,45)$ Mal, präklinisch 8,59 (12,51) Mal. Die Selbsteinschätzung ergab im Median die Note 2. Ibuprofen und Paracetamol, 
welche ebenfalls vom BV-ÄLRD für die Applikation durch Notfallsanitäter vorgeschlagen wurden, verabreichten die Auszubildenden dagegen eher selten. Für Ibuprofen wurden innerklinisch im Schnitt $2,62(7,61)$ Anwendungen und präklinisch $0,41(2,45)$ Anwendungen angegeben. Für Paracetamol innerklinisch 4,31 $(9,29)$ und präklinisch 1,25 $(2,82)$ Gaben. Für Ibuprofen ergab sich in der Selbsteinschätzung im Median eine Note von 4 und für Paracetamol von 3. Metamizol stellt aus dieser Gruppe das potenteste Medikament dar, gleichzeitig kann es insbesondere bei der intravenösen Anwendung zu einer ausgeprägten Hypotonie führen und stellt damit vermutlich auch das Medikament mit der relevantesten unmittelbaren $\mathrm{Ne}$ benwirkung dar. Aufgrund der häufigen Anwendung durch die Auszubildenden muss jedoch davon ausgegangen werden, dass diese mit der Anwendung ausreichend vertraut sind. Welche Rolle Metamizol zukünftig in der präklinischen Versorgung haben wird, bleibt vor dem Hintergrund wiederkehrender Diskussionen um die Nutzen-Risiko-Bewertung und aktueller medikolegaler Fragen bezüglich der Aufklärung über Nebenwirkungen abzuwarten.

Butylscopolamin wurde von den Auszubildenden innerklinisch 2,58 $(4,32)$ und präklinisch 4,03 $(5,54) \mathrm{Mal}$ gegeben. Die Selbsteinschätzung lag bei Note 3. Bei gering ausgeprägtem Nebenwirkungsprofil kann auch hier von einer ausreichenden Erfahrung in der Anwendung ausgegangen werden.

Abzuwarten bleibt, ob und wann eine Markteinführung von Methoxyfluran für den deutschen Markt erfolgt. Die Substanz ist einfach anzuwenden und verfügt über deutliche Vorteile bei der Analgesie von Traumapatienten mit oft wechselnd starken Schmerzen bei Immobilisation und Rettung. Aufgrund der Einfachheit in der Anwendung ist die Substanz damit besonders für eine Gabe durch Notfallsanitäter geeignet und wird z.B. in Australien schon seit Längerem von „Paramedics" eingesetzt. Gleiches gilt prinzipiell für die Anwendung von Lachgas-Sauerstoff-Gemischen, welche insbesondere im britischen Rettungsdienst seit langem etabliert ist. Aufgrund der relativ umständlichen Handhabung und grundsätzlicher pharmakologischer Überlegungen hat sich dies allerdings in Deutschland bis heute nicht durchsetzen können. Der zukünftige Stellenwert dieser Substanzen bleibt daher aktuell unklar.

\section{Benzodiazepine}

Benzodiazepine sollen laut der Empfehlung des BV-ÄLRD bei Krampfanfall bzw. Status epilepticus, zur Sedierung und bei akuten Erregungszuständen von Notfallsanitätern angewendet werden. Die Indikation ist damit relativ unscharf umrissen. Von den Auszubildenden wurden Benzodiazepine sowohl innerklinisch mit 8,20 $(11,36)$ Anwendungen als auch präklinisch mit 7,27 (10,28) Anwendungen insgesamt häufig eingesetzt. Die Selbsteinschätzung der Auszubildenden, bezogen auf die Anwendung der jeweils auf deren Rettungsmittel vorhandenen Benzodiazepine, ergab im Median eine Note von 3. Bei der Gabe von Benzodiazepinen können vereinzelt schwere Nebenwirkungen auftreten. Es bedarf daher einer gewissen klinischen Erfahrung, insbesondere auch bei der Applikation der jeweiligen Substanzen bei Menschen unterschiedlichen Alters und Allgemeinzustands. Die Indikationen für die Anwendung durch Notfallsanitäter sollten daher letztlich klar definiert sein. Aufgrund der relativ häufigen Anwendung von Benzodiazepinen durch die Auszubildenden besteht vermutlich eine ausreichende Expertise, um diese Substanzen - bei gegebener Indikation - in der Regel sicher einsetzen zu können. Trotzdem wäre eine größere klinische Erfahrung, insbesondere bei intravenöser Gabe, aus Sicht der Autoren sinnvoll. Die intranasale oder rektale Applikation stellt, aus pharmakodynamischer Sicht, dagegen vermutlich die unkritischere Applikationsform dar und sollte für Notfallsanitäter daher ggf. primär in Betracht gezogen werden. Die Applikationsform wurde in dieser Untersuchung allerdings nicht erfragt, sodass über die Erfahrungen mit einzelnen Applikationsformen keine Erkenntnisse vorliegen. Die Kombination mit anderen analgetisch oder sedativ wirkenden Substanzen stellt darüber hinaus ein eigenes Problemfeld dar, welches hier nur unzureichend abgebildet wird.
Lidocain und andere

\section{Lokalanästhetika}

Der BV-ÄLRD hat in seinen Maßnahmenkatalog für Notfallsanitäter auch die intraossäre Punktion mit aufgenommen. In diesem Rahmen kann die Betäubung des Periosts sowie das langsame Vorspülen des Zugangs mit Lidocain bei der Anlage notwendig werden. Lidocain wurde daher für die Gabe durch Notfallsanitäter empfohlen. Die Auszubildenden haben Lokalanästhetika innerklinisch 8,30 (12,52) Mal angewendet. Präklinisch wurden diese dagegen nur vereinzelt gegeben, mit $0,28(0,81) \mathrm{An}$ wendungen. Die Gabe einer geringen Menge Lidocain ist insgesamt als unbedenklich zu werten, selbst wenn diese beabsichtigt oder akzidentiell intravasal erfolgt. Ob die Auszubildenden über ausreichend Erfahrungen verfügen, um das Pharmakon im Rahmen der Anlage eines intraossären Zugangs korrekt einzusetzen, kann auf dieser Datenbasis letztlich nicht geschlussfolgert werden. Die schlechte Selbsteinschätzung mit im Median Note 4 weist jedenfalls auf eine deutliche Unsicherheit in der Anwendung hin.

\section{Glukose}

Glukose ist ebenfalls ein Medikament, das bereits seit Langem intravenös durch Rettungsdienstpersonal zur Behandlung der Hypoglykämie angewendet wird. Auch wenn insbesondere die paravasale Injektion mit gravierenden Folgen verbunden ist, zeigt die Erfahrung aus dem Notarztdienst, dass es sich hierbei um eine eher seltene Komplikation handelt. Die Auszubildenden hatten innerklinisch nur selten die Gelegenheit, Glukose zu verabreichen. Im Mittel wurden nur 1,32 $(2,33)$ innerklinische Anwendungen genannt. Präklinisch wurde das Medikament dagegen mit 7,38 $(6,67)$ Anwendungen deutlich häufiger verabreicht. Die Erfahrungen aus dem Rettungs- und Notarztdienst zeigen, dass dieses Medikament durch Rettungsdienstpersonal korrekt und effektiv angewendet werden kann. Die Selbsteinschätzung der Auszubildenden stimmt hiermit ebenfalls überein. Die in der Ausbildung gewonnene Erfahrung mit dem Medikament muss daher als ausreichend betrachtet werden. 


\section{Antidota}

Naloxon und Atropin sind die einzigen Antidota, welche vom BV-ÄLRD für die Gabe durch Notfallsanitäter empfohlen wurden. Atropin wurde dabei bereits für die Gabe durch Rettungsassistenten bei Intoxikation mit Alkylphosphaten durch die Bundesärztekammer empfohlen. Diese Indikation ist allerdings extrem selten und die meisten Rettungswagen verfügen nicht über Atropin in ausreichender Dosierung. Die Anwendung bei Alkylphosphatintoxikation wurde von den Auszubildenden 0,06 $(0,29)$ Mal genannt. Nach einer Selbsteinschätzung wurde hierzu nicht explizit gefragt. Insgesamt wird in den allermeisten Fällen niemand aus dem Rettungsteam - weder ärztlicherseits noch nichtärztlicherseits - über praktische Erfahrungen mit dem Notfallbild verfügen. Von einer sicheren Anwendung im Sinne von „erlernt" und "beherrschen“, wie dies der Gesetzgeber fordert, kann demnach nicht ausgegangen werden. Bei der Behandlung des Patienten nach einem Algorithmus stellt die korrekte Diagnose bzw. Indikationsstellung somit die wesentliche Schwierigkeit dar.

Eine Naloxongabe wurde von den Auszubildenden innerklinisch $1,00(1,93)$ und präklinisch 0,20 $(0,67)$ Mal durchgeführt. Das Medikament wurde damit selten von den Auszubildenden angewendet. Obgleich sich die titrierende Anwendung vermeintlich einfach gestaltet, führte die fehlende Erfahrung der Auszubildenden zu einer entsprechend schlechten Selbsteinschätzung mit im Median Note 4. Da es sich um eine risikoarme, aber lebensrettende Substanz handelt, sollte die Ausbildung hier verbessert werden.

\section{Tranexamsäure}

Tranexamsäure wurde bisher weder vom BV-ÄLRD noch von anderen Fachgesellschaften für die Gabe durch Notfallsanitäter empfohlen. Aufgrund der hohen Relevanz einer möglichst frühzeitigen Gabe bei polytraumatisierten und massiv blutenden Patienten $[10,11]$ schien den Autoren dieser Studie die Frage nach der in der Ausbildung erlangten Expertise trotzdem interessant. Innerklinisch konnten die Auszubildenden die Substanz 2,56
$(10,39)$ Mal anwenden, präklinisch dagegen nur 0,10 $(0,34)$ Mal, was vermutlich der letztlich seltenen Inzidenz entsprechender Einsatzsituationen geschuldet ist $[12,13]$. Die Selbsteinschätzung der Auszubildenden zeigte mit im Median Note 6 gravierende Unsicherheiten bezüglich der Substanz.

\section{Verhältnis von Anzahl durchge- führter Medikamentengaben zu Selbsteinschätzung}

Um zu überprüfen, ob eine größere praktische Erfahrung in der Verabreichung von Medikamenten zu einer besseren Selbsteinschätzung der Auszubildenden führt, wurde für alle Selbsteinschätzungen der Korrelationskoeffizient nach Spearman berechnet. Eine negative Korrelation zwischen Anzahl an Applikationen und Höhe des numerischen Notenwerts würde für eine Verbesserung der Selbsteinschätzung durch eine höhere Erfahrung bzw. Anzahl an Anwendung der Substanzen sprechen. Eine mittlere Korrelation (ab $\left.r_{S}=-0,3\right)$ konnte lediglich für den Einsatz von Antihistaminika präklinisch sowie für Novalgin und Lokalanästhetika innerklinisch gezeigt werden. Eine starke Korrelation $\left(a b r_{s}=-0,5\right)$ konnte für Ipratropium präklinisch sowie für Akrinor ${ }^{\circledR}$ und Ondansetron innerklinisch gezeigt werden.

Die Selbsteinschätzung der Auszubildenden unterliegt verschiedenen Phänomenen, wie beispielsweise dem KrugerDunning-Effekt [14], und ist daher mit Vorsicht zu interpretieren. Zwar führt eine höhere Anzahl an Anwendungen auch zu einer größeren praktischen Erfahrung der Auszubildenden, dies muss sich jedoch nicht zwingend auch in einer besseren Selbsteinschätzung niederschlagen, zum Teil kann sogar das Gegenteil der Fall sein, wie der positive Korrelationskoeffizient für die intravenöse Therapie mit Beta-2-Mimetika sowie für Nitrendipin und Tranexamsäure präklinisch zeigt.

\section{Limitationen}

Aus datenschutzrechtlichen Gründen bestanden seitens der teilnehmenden Schulen Bedenken hinsichtlich einer zunächst geplanten Einsicht in die Praktikumshef- te der Auszubildenden. Es wurde daher die Form eines von den Befragten Auszubildenden selbst auszufüllenden Fragebogens gewählt. Die teilnehmenden Auszubildenden wurden gebeten, die Anzahl der Medikamentengaben soweit möglich aus ihrem Praktikumsheft zu entnehmen und sofern nicht möglich die Anzahl der Medikamentengaben zu schätzen. Insbesondere die Anzahl selten applizierter Medikamente konnte von den Auszubildenden aber sehr exakt angegeben werden, und die präsentierten Daten erscheinen insgesamt plausibel.

Eine Selbsteinschätzung unterliegt Phänomenen, die zu einer Selbstüberschätzung führen können, und ist daher mit Vorsicht zu interpretieren. Dies ist insbesondere für selten durchgeführte Maßnahmen eher zu erwarten [14]. Eine schlechte Selbsteinschätzung kann aus Sicht der Autoren hingegen jedoch gut geeignet sein, um Defizite in der Handlungskompetenz der Auszubildenden aufzuzeigen.

Bei einer durchgehend hohen Standardabweichung muss davon ausgegangen werden, dass es innerhalb der Studienteilnehmer große interindividuelle Unterschiede gab, was die Interpretation der Daten erschwert. Darüber hinaus sind die Ergebnisse der angewendeten statistischen Testverfahren aufgrund o.g. Einschränkungen bei der Erhebung der Primärdaten mit Vorsicht zu interpretieren.

Letztlich beschränkt sich die vorliegende Untersuchung auf drei Rettungsdienstschulen und die Auszubildenden gehörten zu den ersten in Vollzeit ausgebildeten Notfallsanitätern in Niedersachsen. Eine Reevaluation in einem gewissen zeitlichen Abstand erscheint daher sinnvoll.

\section{Bewertung und Implikationen für die Ausbildung zukünftiger Notfallsanitäter}

Derzeit orientiert sich die Ausbildung angehender Notfallsanitäter maßgeblich an den Empfehlungen des BV-ÄLRD zu Maßnahmenkatalog und Medikamentenliste und den daraus entstandenen Algorithmen bzw. Standardarbeitsanweisungen der einzelnen Bundesländer bzw. Ärztlichen Leiter Rettungsdienst. Diese Empfehlungen entstanden, da der Ge- 
setzgeber nicht eindeutig geregelt hat, welche Maßnahmen und Medikamente für den Einsatz durch Notfallsanitäter im Einzelnen infrage kommen. Daraus folgt aber auch, dass Veränderungen und Entwicklungen in der Medizin zeitnah in den Rettungsdienstalltag implementiert werden können, ohne hierzu eine Gesetzesänderung notwendig zu machen. Der Anspruch der Notfallsanitäterausbildung darf demnach nicht nur die Abbildung gegenwärtig empfohlener Medikamente und Maßnahmen als Lernziele der Ausbildung sein. Zumindest für gegenwärtig absehbare Entwicklungen ist zu fordern, dass diese in der Ausbildung ebenfalls entsprechend abgebildet werden.

Die Bewertung, welche Medikamente bzw. Pharmakotherapien von den Notfallsanitätern nach der Ausbildung ausreichend beherrscht werden und letztlich sicher und effektiv von diesen angewendet werden können, ist schwierig. Bezüglich der Abwägung zwischen Risiken und Nutzen, der korrekten Dosierung und der damit verbundenen, notwendigen Erfahrung sind die aktuell diskutierten Medikamente extrem heterogen. Vereinzelt existieren Studien zur Anwendung bestimmter Medikamente durch Notfallsanitäter [6-9]. Für manche, bereits seit Längerem durch Rettungsdienstpersonal eingesetzte Medikamente zeigt die Erfahrung, dass diese sicher, effektiv und mit einer akzeptablen Rate an Komplikationen durch Rettungsdienstpersonal eingesetzt werden können. Studien zu Lernkurven existieren für die Applikation von Medikamenten nicht. Die im Rahmen der vorliegenden Untersuchung erhobene Anzahl von Medikamentenapplikationen kann daher meist nur vor dem Hintergrund klinischer Erfahrung bewertet werden. Weitere Studien zu Lernkurven sind daher dringend notwendig, um ein notwendiges Kompetenzniveau operationalisieren zu können. Die zum Teil hohen Standardabweichungen zeigen an, dass die praktische Ausbildung der Notfallsanitäter sehr unterschiedlich verläuft. Um ein einheitliches Kompetenzniveau am Ende der Ausbildung zu erreichen, scheint es daher notwendig, auch für die Medikamentengabe Mindestanwendungszahlen zu formulieren.

\section{Fazit}

- Auf dem Boden der vorliegenden Daten ergibt sich aus Sicht der Autoren für die meisten der diskutierten Medikamente eine ausreichende Expertise.

- Ketamin erscheint aufgrund deutlich geringerer Erfahrungen weniger geeignet, obgleich gegenwärtig in vielen Algorithmen bzw. Standardarbeitsanweisungen für Notfallsanitäter deutlich favorisiert.

- Für Benzodiazepine erscheint aus Sicht der Autoren eine größere Erfahrung als wünschenswert, insbesondere bei Kombination mit anderen Substanzen und unterschiedlichen Gruppen von Notfallpatienten.

- Für die Gabe von Antiemetika bei Übelkeit bestand in dieser Untersuchung letztlich die höchste Expertise für Ondansetron, welchem auch aufgrund der gering ausgeprägten Nebenwirkungen aus Sicht der Autoren der Vorzug gegeben werden sollte.

- Das vom BV-ÄLRD vorgeschlagene Nitrendipin wurde von den Auszubildenden fast gar nicht angewendet. Bezüglich der Gabe von Urapidil, welches vom BV-ÄLRD jedoch nicht vorgeschlagen wurde, bestand eine deutlich größere Erfahrung seitens der Notfallsanitäter.

- Bezüglich der Gabe von intravenösen Betamimetika, Naloxon und Atropin bei Intoxikationen sowie Lidocain zur Lokalanästhesie bei Anlage eines intraossären Zugangs bestanden bei den Auszubildenden erhebliche Unsicherheiten, sodass die Ausbildung hier verbessert werden muss.

- Die Kompetenzentwicklung im jungen Berufsbild des Notfallsanitäters muss in Rahmen von weiteren Studien und Maßnahmen der kontinuierlichen Qualitätssicherung untersucht und regelmäßig reevaluiert werden.

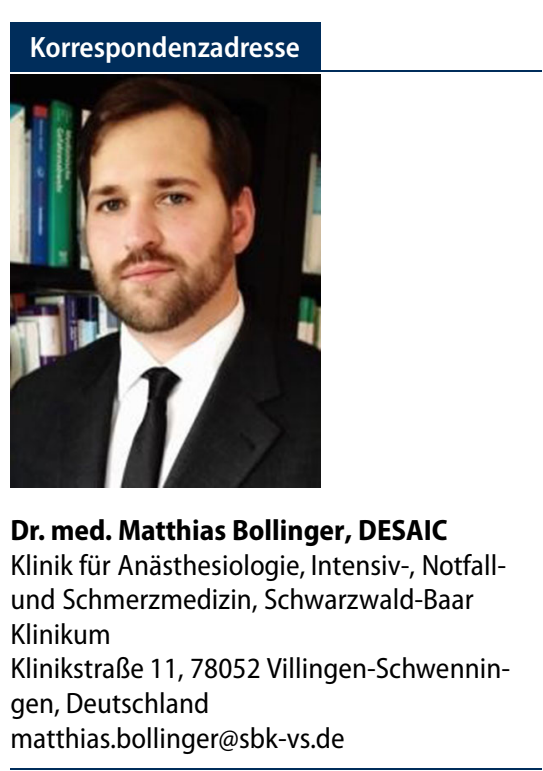

Funding. Open Access funding enabled and organized by Projekt DEAL.

\section{Einhaltung ethischer Richtlinien}

Interessenkonflikt. M. Bollinger und S.G. Russo geben an, dass kein Interessenkonflikt besteht.

Für diesen Beitrag wurden von den Autoren keine Studien an Menschen oder Tieren durchgeführt. Für die aufgeführten Studien gelten die jeweils dort angegebenen ethischen Richtlinien.

Open Access. Dieser Artikel wird unter der Creative Commons Namensnennung 4.0 International Lizenz veröffentlicht, welche die Nutzung, Vervielfältigung, Bearbeitung, Verbreitung und Wiedergabe in jeglichem Medium und Format erlaubt, sofern Sie den/die ursprünglichen Autor(en) und die Quelle ordnungsgemäß nennen, einen Link zur Creative Commons Lizenz beifügen und angeben, ob Änderungen vorgenommen wurden.

Die in diesem Artikel enthaltenen Bilder und sonstiges Drittmaterial unterliegen ebenfalls der genannten Creative Commons Lizenz, sofern sich aus der Abbildungslegende nichts anderes ergibt. Sofern das betreffende Material nicht unter der genannten Creative Commons Lizenz steht und die betreffende Handlung nicht nach gesetzlichen Vorschriften erlaubt ist, ist für die oben aufgeführten Weiterverwendungen des Materials die Einwilligung des jeweiligen Rechteinhabers einzuholen.

Weitere Details zur Lizenz entnehmen Sie bitte der Lizenzinformation auf http://creativecommons.org/ licenses/by/4.0/deed.de.

\section{Literatur}

1. Notfallsanitätergesetz vom 22. Mai 2013 (BGBI. I S. 1348), das durch Artikel $1 \mathrm{~h}$ des Gesetzes vom 4. April 2017 (BGBI. I S. 778) geändert worden ist. (NotSanG) 
2. Lechleuthner A (2014) Invasive Maßnahmen für Notfallsanitäter. Beherrschen, verstehen und sicher anwenden. Rettungsdienst 37(1):14-18

3. Bundesverband Ärztlicher Leiter Rettungsdienst e. V.(2014)Bericht über dieErörterung und Abstimmung zu invasiven Maßnahmen für zukünftige Notfallsanitäterinnen und -sanitäter (Pyramidenprozess). Stellungnahme von 15.02.2014

4. Bollinger M, Langner M, Wellershaus D, Kaisers W, Russo SG (2019) Durchführung invasiver ärztlicher Maßnahmen durch Notfallsanitäter: Eine Studie mit Blick auf die Ausbildung. Notfall Rettungsmed. https://doi.org/10.1007/s10049-019-0591-3

5. Bundesärztekammer (1992) Notfall: Notkompetenz. Stellungnahme der Bundesärztekammer zur Notkompetenz von Rettungsassistenten und zur Delegation ärztlicher Leistungen im Rettungsdienst. http://www.bundesaerztekammer. de/page.asp?his=0.7.47.3222. Zugegriffen: 13 . Jan. 2014

6. Kill C, Greb I, Wranze E et al (2007) Kompetenzentwicklung im Rettungsdienst: Ein Pilotprojekt zur erweiterten Notfalltherapie durch Rettungsassistenten. Notfall Rettungsmed 10:266-272

7. Grebl,Wranze E, Hartmann Het al (2011) Analgesie beim Extremitätentrauma durch Rettungsfachpersonal: Daten zu Sicherheit und Wirksamkeit bei präklinischer Morphingabe. Notfall Rettungsmed 14:135-142

8. Häske D, Schempf B, Gaier G, Niederberger C (2014) Prähospitale Analgosedierung durch Rettungsassistenten: Effektivität und Prozessqualität unter ärztlicher Supervision. Anaesthesist 63:209-216

9. Schempf B, Casu S, Häske D (2017) Prähospitale Analgosedierung durch Notärzte und Rettungsassistenten: Vergleich der Effektivität. Anaesthesist 66:325-332

10. CRASH-2 trial collaborators, Shakur H, Roberts I, Bautista R et al (2010) Effects of tranexamic acid on death, vascular occlusive events, and blood transfusion in trauma patients with significant haemorrhage (CRASH-2): a randomised, placebocontrolled trial. Lancet 376:23-32

11. The CRASH-2 collaborators (2011) The importance of early treatment with tranexamicacid in bleeding trauma patients: an exploratory analysis of the CRASH-2 randomised controlled trial. Lancet 377(9771):1096-1101.e2

12. Gries et al (2005) Einsatzrealität im Notarztdienst. Notfall Rettungsmed 8:391-398

13. Bollinger M, Roessler M, Russo SG (2015) Inzidenz invasiver ärztlicher Maßnahmen im Rettungsdienst - Eine Betrachtung mit Blick auf den Notfallsanitäter. Notfall Rettungsmed 18(3):215-221

14. Kruger J, Dunning D (1999) Unskilled and unaware of it: how difficulties in recognizing one's own incompetence lead to inflated self-assessments. JPers Soc Psychol 77:1121-1134

\section{Administration of drugs by Paramedics in Germany. A study regarding paramedic education}

Background: The German board of EMS medical directors, the "Bundesverband der Ärztlichen Leiter Rettungsdienst", recommended a comprehensive catalogue of drugs that may be administered by paramedics. We evaluated how often paramedic students administered these drugs during their training.

Methods: We asked 71 students how often they administered 30 different drugs during their training. Statistical analysis was performed with SOFA (Paton-Simpson and Associates Ltd, USA). The Results are displayed as mean (SD).

Results: Intravenous epinephrine was administered 2.22 (6.67) times. Atropine was used for treatment of bradycardia 1.18 (1.97) times. Benzodiazepines were administered 7.27 (10.28) times prehospital. Inhaled beta-agonists were given 8.89 (8.06) times, ipratropium bromide 3.70 (5.91) times, steroids 3.79 (5.32) times and antihistamines 1.82 (4.34) times prehospital. Ondansetron was given 14.38 (35.06) times in-hospital. Dimenhydrinate was the most widely used antiemetic in the prehospital environment with 8.14 (10.14) administrations. Furosemide was used 6.06 (9.45) times prehospital. Heparin was used 12.00 (14.32) times, acetylsalicylic acid 14.32 (14.29) times and glyceroltrinitrate 12.25 (10.87) times prehospital. Metamizole was used 8.59 (12.51) times, ketamine 3.51 (5.23) times, opioids 10.72 (16.18) times and naloxone $0.20(0.67)$ times prehospital. Glucose was administered 7.38 (6.67) times prehospital.

Conclusion: It is difficult to evaluate which drugs are suitable for administration by paramedics because of lacking evidence or consensus about an indicated number of administrations. Therefore, an evaluation can only be derived from clinical experience. However from the authors' point of view the paramedics have satisfactory expertise for administering most of the investigated drugs.

\section{Keywords}

Emergency medicine · Board of EMS Medical Directors - Emergency drugs · Invasive procedures . Self-assessment 